\title{
La impostergable invención del presente: Literatura guatemalteca actual
}

\section{Luis Méndez Salinas y Carmen Lucía Alvarado}

\author{
"Tendrá que ser así: \\ voy en mi busca \\ quizá partiendo \\ de algún presente incierto...» \\ Roberto Monzón
}

I.

Nos acercamos al final de la segunda década del siglo XXI, y Guatemala sigue siendo una ficción y una herida. Su pasado es el recuento de las pesadillas más tremendas y su presente parece ser producto de la fragmentación, el sinsentido y la violencia. Guatemala es un cuerpo social dislocado en el que se dibuja un delirio: el arte.

Los guatemaltecos hemos sido testigos de la deformación profunda del cuerpo geográfico, social y espiritual que habitamos, ese cuerpo que ha aprendido a soportar el paso y el peso de la historia como una interminable golpiza que va dejándolo inválido. El primer golpe fue la invasión y la conquista; luego vino la farsa de la «emancipación» y el «progreso», la «libre empresa», las dictaduras, la intervención norteamericana, la intolerancia, el horror y la muerte.

Sin embargo, en medio de ese panorama repleto de patologías, donde todo parece ser posible menos la creación, la libertad y el deseo, han surgido siempre poderosos anticuerpos de luz que rescatan las pocas facultades que le han quedado intactas al cuerpo mutilado de nuestra «nación». Esas facultades son la sensibilidad, la imaginación creadora, la memoria y la conciencia colectiva. Sobre ellas se asienta la rotunda constelación del arte guatemalteco, que es una de las respuestas más potentes que como colectividad hemos dado ante la magnitud del drama que se produce en esta tierra, y que se constituye como un irrenunciable acto de fe en la capacidad creadora del ser humano.

Así pues, por un lado está la horrenda historia política y están sus fragmentos todavía dolorosos. Por otro, aparece el arte, la literatura, la creación. La historia de esa creación, su flujo a través del tiempo, no se determina pasiva y linealmente a partir de esa otra historia (la política, la nefasta), pero participa dentro de su dinámica de negaciones y rupturas, de alaridos y silencios, de obstáculos y fugas.

Hemos dicho ya que Guatemala es un país fundado a partir de un trauma, de una fractura violenta que con el tiempo se ha vuelto más honda, más dolorosa, más evidente. De ahí que pensar en Guatemala como «nación» sea pensar en la distancia que separa sus polos, y en la imposibilidad aparente de integrarlos en su sistema coherente, democrático y plural. Hasta ahora, pensar en nuestra historia es pensar en una interminable lista de proyectos fallidos cargados de imposición y violencia, pero es también pensar en una inagotable resistencia. Gracias a dicha resistencia es que las fallas de origen que constituyen la excluyente estructura de la nacionalidad guatemalteca no han podido evitar el surgi- 
miento de un arte vigoroso, rotundo y digno, que se impone a carencias de todo tipo para elaborar una estructura propia que lo sustente. En muchos casos, los artistas guatemaltecos han debido anteponer el exilio a la imposibilidad, la muerte al silencio, la resistencia a la represión.

En un contexto como el que venimos delineando, parece obvia la imposibilidad de hablar del arte (o de la literatura) en términos de una «tradición». A lo sumo, podremos encontrar elementos asilados que se articulan para configurar el carácter de un quehacer. Así, encontraremos infinitas líneas (obras, tentativas) que se desarrollan y estallan, anulando - aparentemente- ${ }^{-}$sus posibilidades de continuidad histórica. Las grandes obras que se han escrito en este país no pueden verse como eslabones de una secuencia lineal: cada una de ellas permanece por sí misma, en su necesidad y su imponencia, accidentando nuestra geografía literaria y articulándose con las otras a manera de constelación. Eso hace que el panorama histórico de la literatura en Guatemala no tenga una forma fija, sino que se niegue, se reinvente y se dibuje nuevos límites a cada tanto.

\section{II.}

Para situarnos en el contexto social y estético en que se desarrolla la literatura guatemalteca actual es necesario remitirnos a mediados de la década de 1990. Con la firma de la paz, hacia 1996, la sociedad guatemalteca iniciaba un proceso de apertura democrática que se creía fundamental para su desarrollo posterior. Terminaba la guerra y empezaba «lo otro»: la paz, la esperanza, el mañana. Si bien esta coyuntura puntual es el referente inmediato de una serie de valiosos procesos para impulsar la regeneración de un tejido social terriblemente desgarrado, poco duró el entusiasmo y la confianza, pues la violencia de carácter político no cesó del todo.

En el papel, el conflicto armado había concluido, pero en la práctica social fuimos testigos de la democratización de la violencia y de la aparición de complejas estructuras del crimen organizado y el narcotráfico, mientras que la práctica política tomó la máscara del juego democrático para justificar medidas de carácter neoliberal que al día de hoy violentan la integridad de nuestras comunidades y nuestros territorios.

En términos generales, durante la transición entre el siglo XX y el siglo XXI parecen colapsar ciertas formas de la esperanza que alimentó buena parte de la resistencia ante los gobiernos represivos de los años previos, y eso tuvo fuertes repercusiones en los ámbitos estéticos y en las relaciones intergeneracionales de la época. Se abre una grieta entre la
La literatura que surge

en este momento tiene

la conciencia de formarse

dentro de un lenguaje

y un espacio simbólico

en mutación, que

experimenta cambios

radicales en cuanto a sus

registros y sus imaginarios.

literatura de posguerra - esa literatura escrita por las generaciones que experimentaron en carne propia el conflicto armado y vieron desmoronarse sus ideales de lucha- y las nuevas escrituras que aparecen inmediatamente después del conflicto. La retórica de la lucha resulta obsoleta y se devela un horizonte hasta entonces inédito que permite el surgimiento de un arte que aparentemente no tiene la necesidad de ser social y busca plantearse desde la más absoluta libertad. Quizá esta es una tendencia natural de reacción ante el trauma que implicaba la tendencia política del pasado inmediato, sin embargo también fue estimulada por discursos que pretendía desmovilizar políticamente a una generación de creadores en el contexto de la posguerra.

La literatura que surge en este momento tiene la conciencia de formarse dentro de un lenguaje y un espacio simbólico en mutación, que experimenta cambios radicales en cuanto a sus registros y sus imaginarios. Es entonces cuando la capacidad expresiva de los jóvenes ingresa con toda su fuerza para revitalizar la conciencia estética del momento. El cuerpo social guatemalteco se ve y se palpa: se encuentra roto. Los fragmentos de ese cuerpo se reconocen en el arte, también fragmentario, también delirante.

El gesto poético (la actitud y no tanto el texto) es quizá el gran protagonista de la escena literaria guatemalteca de 
finales del siglo XX y principios del siglo XXI. La oralidad, la acción, la importancia de un instante compartido en que la poesía se ilumina. Se busca la poesía en los más diversos soportes: video, performance, arte visual y texto escrito; es difícil precisar fronteras. Esto viene de la mano con una necesidad de retomar los espacios públicos para visibilizar el surgimiento de nuevos códigos y nuevas comunidades que se desplazan por el espacio urbano. ${ }^{2}$

Luego del estallido inicial que puede fecharse entre 1996 y 1998, cuando aparecen dos proyectos decisivos para el movimiento literario de la época (Ediciones Bizarras y Editorial X), los jóvenes escritores empiezan a adquirir una conciencia nueva respecto a la importancia de «lo textual» como registro de sus preocupaciones estéticas. Editoriales y revistas, publicaciones independientes, fanzines y lecturas alternativas serán los marcadores de un cambio drástico en los cánones y las dinámicas de hacer literatura en el país.

La libertad y la fuerza de choque que trajo consigo la generación de guatemaltecos que experimentó esa Guatemala fracturada de inicios del siglo XXI (repleta de violencia, esquizofrenia y consumismo), fue el principal sustento de la nueva generación de escritores, que empezó a producir una serie de obras anómalas, patológicas, delirantes. Usaron (y, en muchos casos, abusaron de) lo escatológico, se vieron influenciados por el cine pulp, el cómic y el lenguaje publicitario, experimentaron con distintos formatos y lograron articular un lenguaje propio donde el gesto se vinculó de tal manera con el texto que fue necesario producir nuevos registros y nuevas dinámicas para interactuar con un público ávido de nuevos estímulos. ${ }^{3}$

Las tendencias estéticas iniciadas alrededor de 1996 tuvieron diversos niveles de vigencia, alcance y duración. El lenguaje responde a un proceso constante de mutaciones y divergencias, y la intención creadora a partir de ese lenguaje siempre cambiante debe cambiar con él. En ese sentido, el objetivo inicial de detonación y choque va depurándose con los años y articula nuevos discursos gracias a la existencia de nuevas condiciones. El estruendo se diluye poco a poco y muchos de los autores que tienen que ver con él empiezan a construir obras alrededor de intenciones estéticas más conscientes, más estructuradas. Esto implica que en los últimos 20 años hemos sido testigos de la construcción de una escena, de su crecimiento y su transformación.

En este apartado hemos hecho referencia a un bloque de escritores nacidos entre 1970 y 1980. Todos empezaron a publicar su obra en fechas cercanas a 1996. Los principales engranajes de su dispositivo literario fueron las Ediciones
Mundo Bizarro (proyecto enfocado en la publicación de poesía, a partir de distintos soportes de factura artesanal) y la Editorial X (que tendió más hacia la narrativa y apostó por vincular el diseño gráfico con la producción textual). Ambas editoriales fueron coordinadas por escritores que, tanto con su trabajo literario como editorial, definieron en mucho los parámetros estéticos de la época: Simón Pedroza y Estuardo Prado, respectivamente. Sus publicaciones habituales, y una extensa lista de proyectos paralelos, generaron un espacio alternativo de gran vitalidad que se sumó a la escena configurada por diversas propuestas editoriales ya establecidas, como F\&G Editores, Revista La Ermita, Editorial Letra Negra, Magna Terra Editores y Ediciones del Pensativo. ${ }^{4}$

A nuestro criterio, ${ }^{5}$ el periodo comprendido entre 1996 y 2005 -en que surgen las formas escriturales aquí descritas - se perfila a partir de libros de poesía como bizarro - poemaensucio- (1996), de Simón Pedroza; Diafragma numérico (1999), de Pablo Bromo; La ciudad de los ahogados (1999) y Encierro y divagación en tres espacios y un anexo (2001), de Maurice Echeverría; Raktas (2002) y Soledadbrother (2003), de Javier Payeras; Los nombres ocultos (2002), de Alan Mills; Lunas sucias (2005), de Eduardo Villalobos; y los proyectos colectivos $\mathrm{Te}^{-}$ rrorismo moral y ético (1998) y Automátika 9mm (2001), en los que participaron Alejandro Marré, Pablo Bromo, Javier Payeras y Simón Pedroza.

En la narrativa, destaca la obra de Francisco Alejandro Méndez ${ }^{6}$-Sobrevivir para contarlo (1999) y Crónicas suburbanas (2001)-; La estética del dolor (1998), El libro negro (2000) y Los amos de la noche (2001), de Estuardo Prado; Este cuerpo aquí -Antidiario I- (1998) y Sala de espera (2001), de Maurice Echeverría; Seis cuentos para fumar (2001), de Byron Quiñónez; El retorno del cangrejo, parte 4 (2001), de Julio Calvo Drago; y la novela Ruido de fondo (2003), de Javier Payeras.

Esta brevísima lista de obras y autores responde a un ejercicio particular de lectura que hemos desarrollado durante los últimos 15 años. Da cuenta de una dinámica cultural que tiene como centro la Ciudad de Guatemala. Sin duda es necesario realizar investigaciones exhaustivas que permitan ampliar el foco e integrar dinámicas similares en otros espacios del país. Llama la atención que dentro del catálogo de las editoriales de la época, es prácticamente nula la presencia de escritoras. ${ }^{7}$

\section{III.}

Hacia mediados de la primera década del siglo XXI, aparecen nuevas voces, nuevas búsquedas, nuevas preocupaciones que en los años inmediatamente precedentes qui- 
zá fueron impensables. Muchos de los escritores y escritoras nacidos en la década de 1980 , y que empiezan a publicar su trabajo hacia el año 2006, intuyen la necesidad de indagar en la historia literaria guatemalteca y, simultáneamente, tender puentes hacia las manifestaciones contemporáneas de la literatura en el ámbito inmediato de América Latina. Ambos movimientos - uno hacia el pasado que se descubre y se asume como propio, y otro que apela a la sincronía y complicidad con escritores coetáneos a lo largo y ancho del idioma- han enriquecido notablemente el carácter de la escritura actual en Guatemala.

Las estéticas fundadas hacia el final de los años 90 y consolidadas a principios del siglo XXI, empiezan a poblarse de nuevas voces y dinámicas que le otorgan diversidad al ecosistema literario en el país. Hemos dicho ya que la primera etapa del proceso iniciado en 1996 predominan las experiencias desarrolladas en el contexto de la Ciudad de Guatemala; sin embargo, hacia el año 2003 inicia de forma paralela e independiente un movimiento de poetas jóvenes en Quetzaltenango. Reunidos en el grupo-taller Ritual, los poetas Martín Díaz Valdés, Carmen Lucía Alvarado, Julio Serrano Echeverría y Marvin García, impulsaron un proceso colectivo de crítica literaria aplicado a sus propias obras, y activaron desde la gestión cultural diversos espacios de lectura e intercambio con escritores de otros ámbitos.

En estos años se establece una nueva etapa de intercambio generacional y geográfico, ya que la escena deja ser exclusivamente capitalina y se generan espacios de diálogo entre escritores de las más diversas edades. Además, las voces femeninas resurgen con mucha fuerza. ${ }^{8}$ Poetas de generaciones más lejanas en el tiempo -como Francisco Morales Santos, Francisco Nájera o Enrique Noriegafueron interlocutores fundamentales para el nuevo bloque generacional que empezaba a perfilarse. Tanto en el centro histórico de la Ciudad de Guatemala, ${ }^{9}$ como en el seno de la conservadora colectividad quetzalteca, los nuevos escritores continuaron luchando por la recuperación del espacio público mediante intervenciones con diversos lenguajes que permitían intercambios entre sí y con poetas extranjeros. Entre 2006 y 2009, las búsquedas de los escritores jóvenes estaban en la escritura, pero también en la voz viva. Lo importante era encontrar estímulos y crear. Para los miembros del grupo Ritual, una de las manifestaciones de esa necesidad que sentían fue la creación del Festival Internacional de Poesía de Quetzaltenango, que a la fecha cuenta con 14 ediciones y es el encuentro poético de mayor prestigio en el país.
Antes de que terminara la primera década del siglo XXI, el circuito literario Guatemala-Quetzaltenango estaba plenamente establecido, y empezó a nutrirse de las discusiones y dinámicas propias de lugares como Comalapa y San Pedro La Laguna, que se constituyeron en sitios importantes para el arte plástico, el arte contemporáneo y la escritura. Asimismo, empezaron a aparecer en la escena poetas de Quiché, Izabal y Totonicapán. Esta dinámica aportó perspectivas y experiencias nuevas a los movimientos estéticos que habían germinado en la década anterior.

El panorama de la narrativa guatemalteca -entre finales de 2005 y 2009- se fortaleció notablemente con el surgimiento de libros como Serenatas al hastío (2007) y Retrato de borracho con país (2008), de Eduardo Juárez; Simplemente una invitada (2006), de Lorena Flores-Moscoso; Las flores (2007), de Denise Phé-Funchal; y Los jueces (2009), de Arnoldo Gálvez Suárez. En la poesía destaca Los magos del crepúsculo (2005), de Wingston González; Casa solitaria (2005), de Rosa Chávez; Breves conversaciones de la psicosis (2006), de Juan Pablo Dardón; Las palabras y los días (2006), de Julio Serrano Echeverría; Esco-p(o)etas Para uNa MuErtE en ver(Sos) B-A...L...A (2006), de Manuel Tzoc; Síncopes (2007), de Alan Mills; Gemido de Huellas (2007), de Sabino Esteban Francisco; y Canto palabra de una pareja de muertos (2009), de Pablo García.

Las publicación de estos y otros libros, el aparecimiento de fanzines colectivos, las muestras de poesía contemporánea en el Centro Histórico de la ciudad capital, las intervenciones del grupo Ritual en Quetzaltenango, el crecimiento del FIPQ, la participación de poetas guatemaltecos en festivales y encuentros de diversos países, y su vinculación en una red cada vez más grande de poetas latinoamericanos trajo consigo una nueva necesidad: dejar evidencia tangible de esos encuentros, de esos hallazgos y del asombro que traían consigo. Eso revitalizó notablemente la producción de libros en Guatemala, permitiendo que surgieran varias iniciativas gestionadas por los propios escritores. Así, para el año 2010 se produce un estallido simultáneo que involucró - por lo menos- a tres proyectos editoriales: Vueltegato Editores, Catafixia Editorial y Mata-Mata Ediciones. Ese año, Catafixia publicó 24 títulos de poesía, Mata-Mata puso en circulación 10 libros, y Vueltegato lanzó 6 más. Progresivamente, surgirán nuevos proyectos editoriales, y algunos consolidarán su catálogo a escala continental.

Entre 2010 y 2012 se consolidan las obras de autores que empezaron a publicar en los años previos, y se agregan obras y autores a través de plataformas editoriales como Catafixia ${ }^{10}$ y Editorial Cultura. ${ }^{11}$ Aparecen los libros de poesía 
Vacíos paralelos (2010), de Gabriel Woltke; No somos los mismos (2010), de Marvin García; Rockstar! (2010), de Julio Prado; Cuentos infantiles (2010) y Quizá este día tampoco sea hoy (2010), de Vania Vargas; Manual del Mundo Paraíso (2010), de Denise Phé-Funchal; Este mal (2010), de Martín Díaz Valdés; Timeless punto 11 (2011), de Alejandro Marré; Sal (2011), de Lorena Flores Moscoso; Frank (2011), de Carlos Meza; Actos de magia (2011), de Julio Serrano Echeverría; Imagen y semejanza (2010) y Poetas astronautas (2012), de Carmen Lucía Alvarado; Claustrofilia (2010), de Marco Valerio Reyes; (si) ...algún día nos haremos luces (2010) y Códex (2012), de Luis Méndez Salinas. En la narrativa, es necesario destacar los libros Satanás cabalga mi alma (2010), de Julio Prado; Génesis y encierro (2011), de Rafael Romero; Eva y el tiempo (2012), de Lorena Flores-Moscoso; y Juego de muñecas (2012), de Francisco Alejandro Méndez.
El contexto político y social en que se desarrollan estas escrituras cambia drásticamente en dos momentos puntuales que marcan un antes y un después. Primero, el 10 mayo de 2013 un tribunal guatemalteco emite sentencia condenatoria por genocidio y delitos contra los deberes de humanidad, luego de un largo y doloroso proceso que puso a Efraín Ríos Montt frente a las víctimas del genocidio perpetrado contra el pueblo Ixil a principios de los años 80. Si bien la sentencia fue revocada por la Corte de Constitucionalidad días después, y aunque el juicio está llevándose a cabo nuevamente (incluso después de la muerte de su principal acusado), este proceso impulsó una toma de conciencia respecto a la historia reciente del país en numerosos sectores sociales, principalmente en los jóvenes que, por primera vez, se enfrentaban cara a cara con los horrores del pasado. Este contexto, según

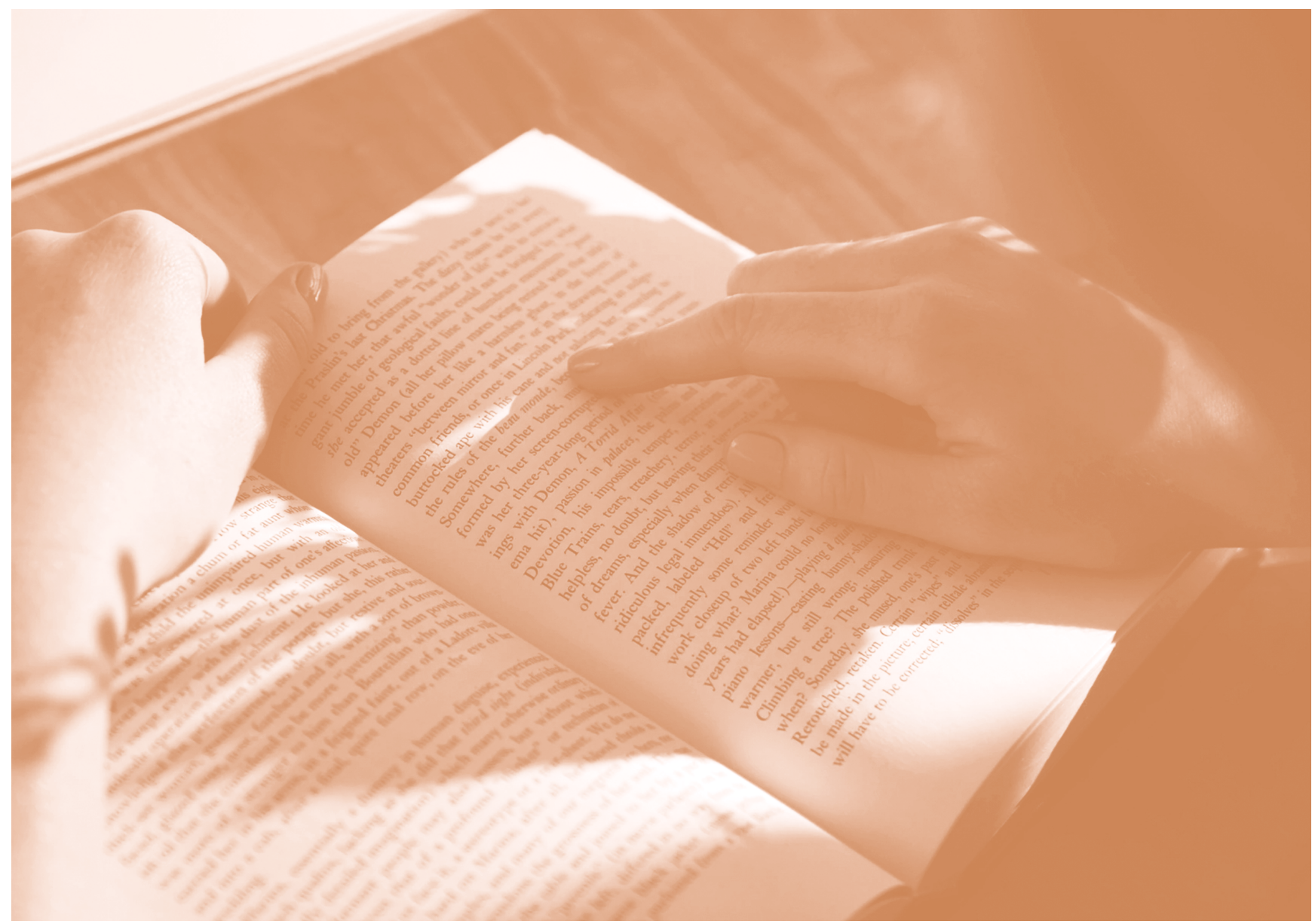


nuestra lectura, determina el fin de la posguerra en Guatemala, y fue la coyuntura propicia para dinamizar una serie de procesos de investigación, reconocimiento y organización social que trasciende las diligencias legales llevadas a cabo.

El segundo momento corresponde a la movilización social masiva que se produjo en el año 2015 a partir de investigaciones desarrolladas por el Ministerio Público y la Comisión Internacional Contra la Impunidad en Guatemala, que destaparon un complejo entramado de corrupción a una escala sin precedentes durante el gobierno de Otto Pérez Molina y Roxana Baldetti Elías (Partido Patriota). Durante meses, cientos de miles de guatemaltecos en todo el país se movilizaron exigiendo la renuncia de la cúpula del poder político, e hicieron que crecieran rápidamente ciertos procesos de organización popular a todo nivel. Si bien los resultados de las elecciones de 2015 fueron nefastos, llevando al poder a una camarilla de militares trasnochados que controlan las decisiones de un presidente-marioneta, diversos movimientos sociales están luchando por articular opciones y propuestas que saquen al país de la severa crisis institucional en que ha estado sumido durante los últimos años.

En ese contexto, la escritura de múltiples autores que pertenecen, incluso, a generaciones distintas- está virando hacia una especie de voz colectiva que da cuenta de la memoria poética del territorio ancestral que habitamos y de la herencia milenaria de nuestra palabra. El arte se vincula de las más diversas vías a posturas políticas pensadas a partir de la resistencia, tomando en cuenta que la estructura que sostiene la producción cultural guatemalteca se sostiene por la voluntad de los mismos artistas, y sin apoyo institucional o económico de ningún tipo. Por otra parte, las sucesivas crisis que atraviesa el país generan la necesidad de buscar y conectar insumos para el conocimiento básico de

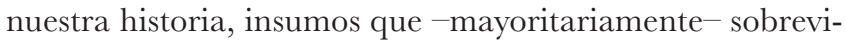
ven en los libros. Esto ha propiciado el fortalecimiento de las iniciativas editoriales existentes, así como el surgimiento de nuevos proyectos con intenciones estéticas y políticas complementarias: Metáfora Editores, La Maleta Ilegal, Proyecto Editorial Los Zopilotes, Sión Editorial, Argot editores, Pequeña Ostuncalco Editorial, Pato/lógica Editores, Alambique, Doble Filo, entre otros, están produciendo sus propios espacios para la difusión de obras literarias de diversa índole.

Es así como escritores nacidos en los años 90, en distintos departamentos del país, empiezan a construir sus propias posibilidades para conjugar la gestión, la edición y la creación. Las voces de autores jóvenes como Julio Cúmez,
Hael López o Luis Morales Rodríguez han encontrado sus caminos digitales e impresos para llegar a un público diverso.

Además, libros esenciales para describir el presente de la literatura guatemalteca están ahora mismo en las manos de su público lector, como sucede con los poemas de Clavícula (2016), de Maurice Echeverría; Constante huida (2016), de Manuel Tzoc; Somos pequeños tiempos (2016), de Pedro Chavajay; Estados de la materia (2017) y Antes del mar (2018), de Julio Serrano Echeverría; Oyonïk (2017), de Julio Cúmez; Raíces de la nostalgia (2017), de Marvin García; Luz de Adán (2018), de Gabriel Woltke; Edad geológica del miedo (2018), de Carmen Lucía Alvarado; y Después de Dios también hay miedo (2018), de Paolo Guinea Ovalle; o con las ficciones de Los mares ignotos (2014), de Carlos Meza; Escolopendra (2014), de Martín Díaz Valdés; Puente adentro (2015), de Arnoldo Gálvez Suárez, Ana sonrie (2015), de Denise Phé-Funchal; Después del fin (2016), de Vania Vargas; Trucha panza arriba (2016), de Rodrigo Fuentes; Pan y cerveza (2017), de Eduardo Halfon; y Trash (2018), de Eduardo Juárez.

La obra de las escritoras y escritores que conforman los bloques generacionales descritos en estas páginas, desde luego, interactúa de distintas maneras con escritores de generaciones previas cuya obra está construyéndose también desde el aquí y desde el ahora. Paradigmáticos son los casos de un pensador como Sergio Valdés Pedroni, poetas como Francisco Morales Santos, Francisco Nájera, Enrique Noriega y Carolina Escobar Sarti, así como de los narradores Rodrigo Rey Rosa, Carol Zardetto, Arturo Arias y Oswaldo Salazar.

IV.

Guatemala, finales de 2018. Cuando se trata de sensibilidad y creación artística, lugar y tiempo parecen ser categorías secundarias. Sin embargo, inventar el presente implica un ajuste de cuentas con el inmenso caudal de voces vivas que nos precedieron. Aquí, con nosotros, en el día a día están Rafael Landívar, José Batres Montúfar y Josefa García Granados; Luis Cardoza y Aragón, Miguel Ángel Asturias, César Brañas y Rafael Arévalo Martínez; Alfonso Orantes, José María López Valdizón y Humberto Hernández Cobos; Otto René Castillo, Roberto Obregón, Luis de Lión y Marco Antonio Flores; Luz Méndez, Margarita Carrera, Antonio Brañas y Roberto Monzón; los del grupo Acento, los del Saker-ti, los de Nuevo Signo. Aquí, con nosotros, en el día a día.

Inventar el presente significa reconocer que la literatura guatemalteca no está formada solo por ciertos textos escritos en español; que es algo que trasciende a la palabra, que 
se funde con la memoria, que habla en distintos registros; que está escrito en papel, en piedra y en la voz; que la literatura guatemalteca es un tronco de incontables ramas que conecta con la profunda raíz ancestral que sostiene nuestra sensibilidad y nuestros símbolos.

Inventar el presente es asumirnos como una red tendida sobre todos los tiempos y cuyo objetivo último es establecer unidades que trasciendan cualquier linealidad posible. He ahí nuestra resistencia: estamos juntos, somos polifonía que fractura el tiempo y el espacio. La red que conforman nuestras palabras y nuestra conciencia convive ya con quienes no nacen aún, pero que asumirán la responsabilidad de la palabra en este territorio. Fundaremos juntos una tradición sin líneas rectas para que, en palabras del poeta Antonio Brañas, «toda esa luz que poseemos no sea, eternamente, un montón de plumas furiosas sobre una aldea dormida».

\section{Notas}

(1) Baste recordar el asesinato de Monseñor Juan Gerardi Conedera, el 26 de abril de 1998, tan solo dos días después de presentar los resultados del proyecto de Recuperación de la Memoria Histórica, que documenta las graves violaciones a los derechos humanos durante la guerra.

(2) Estas dinámicas se refieren particularmente a lo acontecido en la Ciudad de Guatemala y Quetzaltenango, los dos principales centros urbanos del país. Sin embargo, dinámicas particulares y poco estudiadas hasta hoy se desarrollan con mucha fuerza en espacios como Comalapa y San Pedro La Laguna, por mencionar los casos más emblemáticos.

(3) La literatura de esta época surgió en estrecho vínculo con escenas paralelas de arte plástico, música alternativa, danza y teatro. Nuevos lenguajes irrumpen en la conciencia estética de la generación que vio el final de la guerra y aprovechó ciertas libertades que fueron posibles gracias a la apertura democrática de 1996.

(4) Dirigidas, respectivamente, por Raúl Figueroa Sarti, Marco Antonio Flores, Armando Rivera, Gerardo Guinea Diez y Ana María Cofiño.
(5) Dicho criterio sustenta la parte antológica de dos muestras que recogen buena parte de la literatura guatemalteca actual: L. MÉNDEZ SALINAS y C. ALVARADO, (2012). El futuro empezó ayer. Apuesta por las nuevas escrituras de Guatemala, Guatemala, Catafixia Editorial-Unesco; L: MÉNDEZ SALINAS (2015). «Poesía guatemalteca actual». Revista Punto de Partida, n 190.

(6) Galardonado en el año 2017 con el Premio Nacional de Literatura Miguel Ángel Asturias, máximo galardón que otorga el Estado guatemalteco a la trayectoria literaria.

(7) En Editorial X, por ejemplo, únicamente se publicó la antología femenina Hijas de Shakti (1998), coordinada por Nancy Quiñónez; así como el libro Felicidad doméstica y otras cosas aterradoras (2002), de la salvadoreña Jacinta Escudos.

(8) Retomando, de diversas maneras, el espacio que en décadas anteriores habían ocupado poetas fundamentales como Isabel de los Ángeles Ruano, Delia Quiñónez, Luz Méndez de la Vega, Margarita Carrera o Ana María Rodas, entre otras.

(9) A través del proyecto Folio 114, impulsado por Simón Pedroza y heredero de dinámicas colectivas iniciadas en la década anterior, como Casa Bizarra.

(10) A la fecha, Catafixia Editorial ha publicado 99 títulos, incluyendo autores de 15 países de habla hispana. Integran su catálogo voces canónicas de la poesía actual (como Raúl Zurita, Antonio Gamoneda, Francisco Nájera, Caballero Bonald, Enrique Verástegui, Isabel de los Ángeles Ruano, Paula Ilabaca Núñez, entre otros) y poetas jóvenes de Iberoamérica.

(11) Proyecto estatal, coordinado por el poeta Francisco Morales Santos. Editorial Cultura ha desempeñado un papel fundamental para dar a conocer nuevas voces dentro de la literatura guatemalteca durante los últimos 20 años, pese a enfrentar carencias de todo tipo. 